\title{
HEREDITARILY ASPHERICAL COMPACTA
}

\author{
JERZY DYDAK AND KATSUYA YOKOI
}

(Communicated by James West)

\begin{abstract}
The notion of (strongly) hereditarily aspherical compacta introduced by Daverman (1991) is modified. The main results are:

Theorem. If $X \in L C^{1}$ is a hereditarily aspherical compactum, then $X \in$ ANR. In particular, $X$ is strongly hereditarily aspherical.

Theorem. Suppose $f: X \rightarrow Y$ is a cell-like map of compacta and $f^{-1}(A)$ is shape aspherical for each closed subset $A$ of $Y$. Then

1. $Y$ is hereditarily shape aspherical,

2. $f$ is a hereditary shape equivalence,

3. $\operatorname{dim} X \geq \operatorname{dim} Y$.

Theorem. Suppose $G$ is a group containing integers. Then the following conditions are equivalent:

1. $\operatorname{dim} X \leq 2$ and $\operatorname{dim}_{G} X=1$,

2. $\operatorname{dim}_{G * \mathbf{z} G} X=1$.

Theorem. Suppose $G$ is a group containing integers. If $\operatorname{dim} X \leq 2$ and $\operatorname{dim}_{G} X=1$, then $X$ is hereditarily shape aspherical.

Theorem. Let $X$ be a two-dimensional, locally connected and semilocally simply connected compactum. Then, for any compactum $Y$

$$
\operatorname{dim}(X \times Y)=\operatorname{dim} X+\operatorname{dim} Y .
$$
\end{abstract}

\section{INTRODUCTION}

Daverman $\left[\mathrm{Da}_{2}\right]$ introduced the notion of (strongly) hereditarily aspherical compacta, which is a natural extension of the usual asphericity. A compactum is hereditarily aspherical if each closed subset $A$ is $k-U V$ for all $k>1$. Saying $A$ is $k-U V$ means that under some embedding of $A$ in the Hilbert cube, each neighborhood $U$ of $A$ contains a neighborhood $V$ of $A$ such that all maps $S^{k} \rightarrow V$ extend to maps $B^{k+1} \rightarrow U$. Of course, this $k-U V$ property is independent of the embedding of $A$ in metrizable ANR's. A compactum $X$ is said to be strongly hereditarily aspherical if $X$ can be embedded in the Hilbert cube $Q$ such that for each $\varepsilon>0$ there exists an $\varepsilon$-cover $\mathcal{U}$ of $X$ by open collection of $Q$, where the union of any subcollection of elements of $\mathcal{U}$ is aspherical. Note that strongly hereditary asphericity implies

Received by the editors April 6, 1994 and, in revised form, November 19, 1994.

1991 Mathematics Subject Classification. Primary 55M10, 54F45.

Key words and phrases. Dimension, cohomological dimension, aspherical compacta, ANR's, absolute extensors, cell-like maps. 
hereditary asphericity. Manifolds and complexes of dimension 3 and higher cannot possess such a hereditary property. Surprisingly, Daverman $\left[\mathrm{Da}_{2}\right]$ constructed examples of strongly hereditarily aspherical 3- and 4-dimensional compacta and hereditarily aspherical generalized 3-dimensional manifold. Recently, Daverman and Dranishnikov [D-D] proved the existence of strongly hereditarily aspherical compacta of arbitrary dimension. Furthermore, Daverman $\left[\mathrm{Da}_{2}\right]$ proved that the class of strongly hereditarily aspherical compacta contains all 2-dimensional compacta of rational cohomological dimension 1 and that cell-like maps defined either on strongly hereditarily aspherical compacta or on locally simply connected hereditarily aspherical compacta cannot raise dimension.

This raises the question of relating the class of locally simply connected hereditarily aspherical compacta to the class of strongly hereditarily aspherical compacta. A natural guess is that the former is included in the latter. In this paper we answer this question positively. Actually, a stronger result holds; locally simply connected hereditarily aspherical compacta are ANR's. In the process of working on that question we were led to a different generalization of asphericity: let us call a compactum $X$ shape aspherical if any map $f: X \rightarrow P$ from $X$ to a polyhedron $P$ factors up to homotopy as $f \approx h g$, where $g: X \rightarrow K, h: K \rightarrow P$ and $K$ is an aspherical CW complex (i.e., $K$ is a $K(G, 1)$ ). Obviously, shape aspherical compacta are aspherical and strongly hereditarily aspherical compacta are hereditarily shape aspherical. We do not know if hereditarily shape aspherical compacta must be strongly hereditarily aspherical but we were able to generalize the following results known to be true for the class of strongly hereditarily aspherical compacta:

Theorem (Daverman-Dranishnikov [D-D]). If $f: X \rightarrow Y$ is cell-like and $f^{-1}(A)$ is a strongly hereditarily aspherical compactum for each closed subset $A$ of $Y$, then $\operatorname{dim} X \geq \operatorname{dim} Y$.

Theorem (Daverman $\left[\mathrm{Da}_{2}\right]$ ). If $\operatorname{dim} X \leq 2$ and $\operatorname{dim}_{\mathbf{Q}} X=1$, then $X$ is strongly hereditarily aspherical.

Theorem (Schepin $\left.\left[\mathrm{Da}_{2}\right]\right)$. If $f: X \rightarrow Y$ is cell-like and $X \in L C^{1}$ is a hereditarily aspherical compactum, then $\operatorname{dim} X \geq \operatorname{dim} Y$.

The following are the main results of the paper:

Theorem. If $X \in L C^{1}$ is a hereditarily aspherical compactum, then $X \in A N R$. In particular, $X$ is strongly hereditarily aspherical.

Theorem. Suppose $f: X \rightarrow Y$ is a cell-like map of compacta and $f^{-1}(A)$ is shape aspherical for each closed subset $A$ of $Y$. Then

1. $Y$ is hereditarily shape aspherical,

2. $f$ is a hereditary shape equivalence,

3. $\operatorname{dim} X \geq \operatorname{dim} Y$.

Theorem. Suppose $G$ is a group containing integers. Then the following conditions are equivalent:

1. $\operatorname{dim} X \leq 2$ and $\operatorname{dim}_{G} X=1$,

2. $\operatorname{dim}_{G * \mathbf{z} G} X=1$, where $* \mathbf{z}$ means the amalgamated free product with $\mathbf{Z}$. 
Theorem. Suppose $G$ is a group containing integers. If $\operatorname{dim} X \leq 2$ and $\operatorname{dim}_{G} X=$ 1 , then $X$ is hereditarily shape aspherical.

In this paper we use the following definition of cohomological dimension: the cohomological dimension of a space $X$ with respect to a coefficient group $G$ is less than or equal to $n$ (denoted by $\operatorname{dim}_{G} X \leq n$ ) provided that every map $f: A \rightarrow$ $K(G, n)$ of a closed subset $A$ of $X$ into an Eilenberg-MacLane space $K(G, n)$ of type $(G, n)$ admits a continuous extension over $X$. If $n>1$, then we require that $G$ is abelian. By the dimension of a space $X$ (denoted by $\operatorname{dim} X$ ) we mean the covering dimension of $X . \mathbf{Z}$ is the additive group of all integers and $\mathbf{Q}$ is the additive group of all rational numbers. The authors would like to express their gratitude to Professors Daverman, Dranishnikov, Engelking and Koyama for their help during preparation of the paper.

\section{Hereditarily aspherical $L C^{1}$ COMPACTA}

Theorem 2.1. If $X \in L C^{1}$ is a hereditarily aspherical compactum, then $X \in$ ANR. In particular, $X$ is strongly hereditarily aspherical.

Proof. First, let us show that $X \in L C^{n}$ for each $n>1$. Assume $X \in L C^{k}$ for some $k>0$. We need to prove that $X \in L C^{k+1}$. According to Theorem 3.2 of $\left[\mathrm{D}_{-} \mathrm{S}_{2}\right]$ it suffices to show that for each $x \in X$ and for each neighborhood $U$ of $x$ there is a neighborhood $V$ of $x$ in $U$ such that the composition of homomorphisms

$$
\pi_{k+1}(V, x) \rightarrow H_{k+1}(V) \rightarrow \check{H}_{k+1}(V) \rightarrow \check{H}_{k+1}(V)
$$

is trivial. Since $\pi_{k+1}(V, x) \rightarrow \check{H}_{k+1}(V)$ factors as $\pi_{k+1}(V, x) \rightarrow \check{\pi}_{k+1}(V) \rightarrow$ $\check{H}_{k+1}(V)$ and $\check{\pi}_{k+1}(V)=0$ for any $V \in U V^{k+1}$, we can choose $V=U$.

Second, let us show that $\pi_{n}(U)=0$ for all open subsets $U$ of $X$ and all $n>1$. Suppose $g: S^{n} \rightarrow U$ and let $Z=g\left(S^{n}\right)$. Since $Z$ is aspherical, it follows from [Dy, Theorem 8.7 on p.41] that $g$ is null-homotopic in $U$.

Now, let us prove $X \in A N R$. By Lefschetz Theorem (see $\left[\mathrm{Bo}_{2}\right]$, Theorem 8.1 on p.112 or $[\mathrm{Hu}]$, Theorem 4.1 on p.122) we have to find, for each $\epsilon>0$, a number $\delta>0$ such that any partial $\delta$-realization of a finite simplicial complex $K$ extends to a full $\epsilon$-realization. Here, a partial (full) $\mu$-realization of $K$ is a map $g:|L| \rightarrow X$, where $K>L>K^{(0)}(L=K)$, such that $\operatorname{diam} g(|\Delta| \cap|L|)<\mu$ for all simplices $\Delta \in K$. By Theorem 4.1 of $[\mathrm{Hu}]$ (p.156) there is a number $\delta>0$ such that given a partial $\delta$-realization $g:|L| \rightarrow X$ of $K$, it extends to a partial $\epsilon$-realization $G:\left|L^{\prime}\right| \rightarrow X$ of $K$, where $K^{(3)}<L^{\prime}$. We may assume that $L^{\prime}$ is a maximal subcomplex of $K$ with that property. If $L^{\prime} \neq K$, then there is a simplex $\Delta \in K \backslash L^{\prime}$ such that $\partial \Delta<L^{\prime}$. Let $m=\operatorname{dim} \Delta$ and $\eta=\max \left\{\operatorname{diam} G\left(\left|s \cap L^{\prime}\right|\right) \mid s \in K\right.$ and $\left.s \succ \Delta\right\}$. Since $\eta<\epsilon$ and the $(m-1)$-homotopy group of the $(\epsilon-\eta) / 2$-neighborhood of $G(|\partial \Delta|)$ is trivial, $G$ extends to a partial $\epsilon$-realization $G^{\prime}:\left|L^{\prime} \cup \Delta\right| \rightarrow X$ of $K$, a contradiction. Thus, $L^{\prime}=K$ which completes the proof of $X \in A N R$.

To prove that $X$ is strongly hereditarily aspherical consider the copy $X \times 0$ of $X$ in $C(X) \times Q$, where $C(X)$ is the cone of $X$. Given $\epsilon>0$ choose a contractible neighborhood $U$ of $0 \in Q$ with $\operatorname{diam} U<\epsilon / 2$. Cover $X$ with open sets $V_{i}, i=$ $1, \ldots, k$, of diameter less than $\epsilon / 2$. Since $V \times U$ is aspherical for any open subset $V$ of $X$, we are done. 
Remark 2.2. Theorem 2.1 gives a partial answer to Question 3 in $\left[\mathrm{Da}_{2}\right]$. Since the property of the hereditary asphericity and of being $L C^{1}$ are preserved on a cell-like map, Theorem 9 in $\left[\mathrm{Da}_{2}\right]$ follows from Theorem 2.1.

Remark 2.3. Daverman $\left[\mathrm{Da}_{2}\right]$ constructed a hereditarily aspherical generalized 3dimensional manifold. Does there exists a hereditarily aspherical ANR of dimension greater than 3 ?

\section{Hereditarily Shape ASPherical COMPACTA}

Definition. A compactum $X$ is shape aspherical if any map $f: X \rightarrow P$ from $X$ to a polyhedron $P$ factors up to homotopy as $f \approx h g$, where $g: X \rightarrow K, h: K \rightarrow P$ and $K$ is an aspherical polyhedron (i.e., $K$ is a $K(G, 1)$ ).

Lemma 3.1. If $X$ is a shape aspherical compactum, then any map $f: X \rightarrow P$ from $X$ to a polyhedron $P$ factors up to homotopy as $f \approx h g$, where $g: X \rightarrow K$, $h: K \rightarrow P$ and $K$ is a countable, locally finite aspherical polyhedron.

Proof. Factor $f$ as $f \approx h^{\prime} g^{\prime}$, where $g^{\prime}: X \rightarrow K^{\prime}, h^{\prime}: K^{\prime} \rightarrow P$ and $K^{\prime}$ is an aspherical polyhedron with the CW topology. Let $G=\pi_{1}\left(K^{\prime}\right)$. Let $N$ be the simplicial complex whose set of vertices is the family of all finitely generated subgroups of $G$ and $\left\langle G_{0}, \ldots, G_{m}\right\rangle$ is an $m$-simplex of $N$ iff $G_{0}>\ldots>G_{m}$. By Lemma 2.6 of [Dy 2 ] we may assume that there is a map $\pi: K^{\prime} \rightarrow N$ such that

1. $\pi^{-1}(\Delta)$ is a subcomplex of $K^{\prime}$ homotopy equivalent to $K\left(G_{0}, 1\right)$ for any simplex $\Delta=\left\langle G_{0}, \ldots, G_{m}\right\rangle$ of $N$.

2. The inclusion $\pi^{-1}(\Delta) \hookrightarrow K^{\prime}$ induces the inclusion $G_{0} \hookrightarrow G$ of the fundamental groups.

Let $L$ be a finite subcomplex of $N$ containing $g^{\prime}(X)$ and let $H$ be the subgroup of $G$ generated by the vertices of $L$. Notice that the join $L^{\prime}=\langle H\rangle * L$ is a subcomplex of $N$ and $\pi^{-1}\left(L^{\prime}\right)$ of the type $K(H, 1)$. Indeed, let $R$ be the family of subcomplexes $S$ of $L$ with the property that $\pi^{-1}(\langle H\rangle) \hookrightarrow \pi^{-1}\left(\langle H\rangle * S^{\prime}\right)$ is a homotopy equivalence for all subcomplexes $S^{\prime}$ of $S$. If $S, T \in R$, then $S \cup T \in R$. Indeed, if $S^{\prime}<S$ and $T^{\prime}<T$, then $\pi^{-1}(\langle H\rangle)$ is a strong deformation retract of both $\pi^{-1}\left(\langle H\rangle * S^{\prime}\right)$ and $\pi^{-1}\left(\langle H\rangle *\left(S^{\prime} \cap T^{\prime}\right)\right)$. Therefore, $\pi^{-1}\left(\langle H\rangle *\left(S^{\prime} \cap T^{\prime}\right)\right)$ is a strong deformation retract of $\pi^{-1}\left(\langle H\rangle * S^{\prime}\right)$ which implies that $\pi^{-1}\left(\langle H\rangle * T^{\prime}\right)$ is a strong deformation retract of $\pi^{-1}\left(\langle H\rangle *\left(S^{\prime} \cup T^{\prime}\right)\right)$.

By induction one can easily prove that all subcomplexes of $L$ belong to $R$. Since $\operatorname{Im} g^{\prime} \subset \pi^{-1}\left(L^{\prime}\right)$, we may replace $\pi^{-1}\left(L^{\prime}\right)$ by a locally finite countable polyhedron. Indeed, $\pi^{-1}\left(L^{\prime}\right)$ is homotopy equivalent to a countable polyhedron $A$ which is homotopy equivalent to $\bigcup_{k=1}^{\infty} A_{k} \times[k-1, k]$, where $A_{1} \subset \ldots \subset A_{k} \subset \ldots$ are finite subcomplexes of $A$ whose union is $A$.

Lemma 3.2. If $X$ is a shape aspherical compactum embedded in the Hilbert cube $Q$ as a $Z$-set, then $X$ has a basis of open neighborhoods consisting of aspherical sets.

Proof. Let $U$ be an open neighborhood of $X$ in $Q$. Let $K$ be an aspherical ANR such that the inclusion $X \hookrightarrow U$ factors as $g f, f: X \rightarrow K, g: K \rightarrow U$. We may assume that $K$ is a separable $Q$-manifold (use Lemma 3.1 and the fact that $K \times Q$ is a separable $Q$-manifold homotopy equivalent to $K$ if $K$ is a locally finite, countable polyhedron), and we may assume that $f$ is a homeomorphism onto a 
$Z$-set $f(X)$ of $K$ (see $[\mathrm{C}]$ p.27) and $g\left|f(X)=i f^{-1}\right| f(X)$. By Theorem 18.2 of [C] (p.27) $g: K \rightarrow U$ can be approximated rel. $f(X)$ by a $Z$-embedding. Thus, assume that $g$ is a $Z$-embedding. By Theorem 16.2 of $[\mathrm{C}]($ p.25) $g(K)$ is collared in $U$. That collar is an open neighborhood of $X$ in $U$ of the homotopy type of $K$. $\square$

Lemma 3.3. If $f: X \rightarrow Y$ is a cell-like map of compacta, $A$ is a closed subset of $Y$ and $g: X \cup M\left(f \mid f^{-1}(A)\right) \rightarrow P$ is a map into an aspherical $A N R$, then $g$ extends over the mapping cylinder $M(f)$ of $f$.

Proof. It is proved in [Wa] (p.117) that for any cell-like map $g: R \rightarrow S$ of compacta and for any aspherical $P \in A N R, g^{*}:[S, P] \rightarrow[R, P]$ is a bijection. Consider inclusions $i: X \times 0 \hookrightarrow X \times 0 \cup f^{-1}(A) \times I, j: X \times 0 \cup f^{-1}(A) \times I \hookrightarrow X \times I$, $k: X \cup M\left(f \mid f^{-1}(A)\right) \rightarrow M(f)$ and cell-like maps $F: X \times I \rightarrow M(f)$ and $H:$ $X \times 0 \cup f^{-1}(A) \times I \rightarrow X \cup M\left(f \mid f^{-1}(A)\right)$ induced by $f$. Since $i^{*}, j^{*}, F^{*}$ and $H^{*}$ are bijections ( $i$ and $j i$ are homotopy equivalences), then $k^{*}$ is a bijection as $k H=F j$.

Theorem 3.4. Suppose $f: X \rightarrow Y$ is a cell-like map of compacta and $f^{-1}(A)$ is shape aspherical for each closed subset $A$ of $Y$. Then

1. $Y$ is hereditarily shape aspherical,

2. $f$ is a hereditary shape equivalence,

3. $\operatorname{dim} X \geq \operatorname{dim} Y$.

Proof. To prove (1) it suffices to show that $Y$ is shape aspherical. Suppose $\pi: Y \rightarrow$ $|K|$ is a map and $K$ is a finite simplicial complex. We may assume that diameters of simplices of $K$ are so small that there is a number $\epsilon>0$ such that any two maps into $|K|$ which are $2 \epsilon$ close are homotopic and $\sup \{\operatorname{diam}|\Delta| \mid \Delta \in K\}<\epsilon$. Extend $\pi f$ to $F: U_{K} \rightarrow|K|$, where $U_{K}$ is an aspherical neighborhood of $X$ in $Q$. Order all subcomplexes of $K$ as $L_{1}=K, L_{2}, \ldots, L_{m}$ in such a way that $L_{i}$ is not a subcomplex of $L_{j}$ if $j>i$. For each $i>1$ let $V_{L_{i}}$ be the $\epsilon$-neighborhood of $\left|L_{i}\right|$ in $|K|$. By induction on $i$ find an aspherical open neighborhood $U_{L_{i}}$ of $f^{-1} \pi^{-1}\left(\left|L_{i}\right|\right)$ in $F^{-1}\left(V_{L_{i}}\right)$ such that if $L_{i}<L_{j}$, then $U_{L_{i}} \subset U_{L_{j}}$. Let $L$ be a maximal subcomplex of $K$ such that there is a map $g_{L}: M\left(f \mid f^{-1} \pi^{-1}(|L|)\right) \rightarrow U_{L}$ with the following properties:

1. $g_{L} \mid f^{-1} \pi^{-1}(|L|)$ is the inclusion.

2. $g_{L}\left(M\left(f \mid f^{-1} \pi^{-1}(|R|)\right) \subset U_{R}\right.$ for all subcomplexes $R$ of $L$.

We claim that $L=K$. Otherwise there is a simplex $\Delta$ in $K \backslash L$ such that $\partial \Delta<L$ and $g_{L} \mid f^{-1} \pi^{-1}(|\partial \Delta|) \subset U_{\partial \Delta}$. By Lemma 3.3, $g_{L}$ extends to $g_{T}$ : $M\left(f \mid f^{-1} \pi^{-1}(|T|)\right) \rightarrow U_{T}$, where $T=L \cup \Delta$. This contradicts maximality of $L$. Now, $F g_{K} \mid Y$ is $2 \epsilon$-close to $\pi$ which proves that $Y$ is shape aspherical.

To prove (2) it suffices to show that $f$ is a shape equivalence, i.e. $f^{*}:[Y, P] \rightarrow$ $[X, P]$ is a bijection for any $P \in A N R$. Given $g: X \rightarrow P$ we can factor it as $g \approx u d$, where $d: X \rightarrow K$ and $K$ is an aspherical polyhedron. It is proved in [Wa] (p.117) that $f^{*}:[Y, K] \rightarrow[X, K]$ is a bijection. In particular, there is $d^{\prime}: Y \rightarrow K$ with $d^{\prime} f \approx d$. Now, $\left(u d^{\prime}\right) f \approx u d \approx g$ which proves that $f^{*}$ is surjective. Suppose $g, g^{\prime}: Y \rightarrow P$ and $g f \approx g^{\prime} f$. Assume $X, Y$ are $Z$-sets in $Q$ and $g, g^{\prime}$ extend to $G, G^{\prime}: U \rightarrow P$ for some aspherical neighborhood $U$ of $Y$ in $Q$ (see Lemma 3.2). We can extend $f: X \rightarrow Y$ to $F: V \rightarrow U$, where $V$ is an aspherical neighborhood of $X$ in $Q$ (see Lemma 3.2) such that $G F \approx G^{\prime} F$. Since $f^{*}:[Y, V] \rightarrow[X, V]$ is a bijection, there is $u: Y \rightarrow V$ such that $u f$ is homotopic to the inclusion 
$X \hookrightarrow V$. Now, $F u f \approx f$ in $U$, so $F u$ is homotopic to the inclusion $Y \hookrightarrow U$. Finally $g \approx G F u \approx G^{\prime} F u \approx g^{\prime}$.

Suppose $\operatorname{dim} X=n$ and $g: A \rightarrow S^{n}$ is a map, where $A$ is a closed subset of $Y$. Let $h: X \rightarrow S^{n}$ be an extension of $g f \mid f^{-1}(A)$. Since $f$ is a shape equivalence, there is $G: Y \rightarrow S^{n}$ such that $G f \approx h$. Notice that $G$ is an extension of $g$ up to homotopy. Indeed, $G f\left|f^{-1}(A) \approx h\right| f^{-1}(A)=g f \mid f^{-1}(A)$ and $f \mid f^{-1}(A)$ is a shape equivalence.

Theorem 3.5. Suppose $G$ is a group containing integers. If $\operatorname{dim} X \leq 2$ and $\operatorname{dim}_{G} X=1$, then $X$ is hereditarily shape aspherical.

Proof. Let us fix a CW complex $K$ containing $S^{1}$ such that $K$ is a $K(G, 1)$ and $\pi_{1}\left(S^{1}\right) \rightarrow \pi_{1}(K)$ corresponds to the inclusion $\mathbf{Z} \hookrightarrow G$. Suppose $f: X \rightarrow L$ is a map and $L$ is a finite simplicial complex. Since $\operatorname{dim} X \leq 2$, we may assume $f(X) \subset L^{(2)}$. For each 2-simplex $\Delta$ of $L^{(2)}$ remove a small disk in the interior of $\Delta$ and attach a copy $K_{\Delta}$ of $K$ along the boundary of the disk. The resulting CW complex $C$ is aspherical (use Lemma 4 of $\left[\mathrm{Da}_{2}\right]$ ) and there is a map $\pi: C \rightarrow L^{(2)}$ obtained by contracting each $K_{\Delta}$ to a point (the barycenter of $\Delta$ ). The fundamental property of this map is that $\pi^{-1}(\Delta) \in A E(X)$ for all simplices $\Delta$ of $L^{(2)}$. Now, Theorem 2.3 of $\left[\mathrm{Dy}_{2}\right]$ says that $f$ factors as $\pi g$ up to homotopy.

Theorem 3.6. Suppose $G$ is a group containing integers. Then the following conditions are equivalent:

1. $\operatorname{dim} X \leq 2$ and $\operatorname{dim}_{G} X=1$,

2. $\operatorname{dim}_{G * \mathbf{z} G} X=1$, where $* \mathbf{z}$ means the amalgamated free product with $\mathbf{Z}$.

Proof. Let us fix a CW complex $K$ containing $S^{1}$ such that $K$ is a $K(G, 1)$ and $\pi_{1}\left(S^{1}\right) \rightarrow \pi_{1}(K)$ corresponds to the inclusion $\mathbf{Z} \hookrightarrow G$. Let $L=K_{0} \cup S^{1} \times[0,1] \cup K_{1}$, where $K_{i}$ is a copy of $K$ containing $S^{1} \times\{i\}$. Notice that $L$ is a $K(G * \mathbf{z} G, 1)$. Let $\pi: L \rightarrow S^{2}$ be the map obtained by contracting $K_{0}$ to the North Pole and $K_{1}$ to the South Pole. Let $U$ be the upper hemisphere of $S^{2}$ and let $D$ be the lower hemisphere of $S^{2}$.

Suppose $\operatorname{dim} X \leq 2, \operatorname{dim}_{G} X=1$ and $f: A \rightarrow L$ is a map, where $A$ is a closed subset of $X$. Let $F: X \rightarrow S^{2}$ be an extension of $\pi f$. Notice that $f$ can be extended to $f^{\prime}: B=A \cup F^{-1}(U \cap D) \rightarrow L$. Since $\pi^{-1}(U) \in K(G, 1)$, there is an extension $f_{0}: F^{-1}(U) \rightarrow \pi^{-1}(U)$ of $f^{\prime}$. Since $\pi^{-1}(D) \in K(G, 1)$, there is an extension $f_{1}: F^{-1}(D) \rightarrow \pi^{-1}(D)$ of $f^{\prime}$. Pasting $f_{0}$ and $f_{1}$ one gets an extension of $f$.

Suppose $\operatorname{dim}_{G * \mathbf{z} G} X=1$. Since $\pi^{-1}(U) \in K(G, 1)$ is a retract of $L$, we get $\operatorname{dim}_{G} X=1$. Suppose $f: A \rightarrow S^{2}$ is a map, where $A$ is a closed subset of $X$. Let $A^{\prime}=f^{-1}(U \cap D)$. Extend $\pi^{-1} f \mid A^{\prime}$ to $g_{0}: f^{-1}(U) \rightarrow \pi^{-1}(U)$ and extend $\pi^{-1} f \mid A^{\prime}$ to $g_{1}: f^{-1}(D) \rightarrow \pi^{-1}(D)$. Paste $g_{0}$ and $g_{1}$ to get $g: A \rightarrow L$ which extends to $G: X \rightarrow L$. Notice that $\pi G$ is a homotopy extension of $f$.

Definition. We say that a compactum $X$ is of perfect cohomological dimension 1 provided $\operatorname{dim}_{G} X=1$ for all perfect groups $G$.

Remark. Compacta of perfect cohomological dimension 1 were introduced in [D-R] as Kainian compacta.

Corollary 3.7 (Dranishnikov-Repovš $[\mathrm{D}-\mathrm{R}]$ ). If $X$ is a compactum of perfect cohomological dimension 1, then $\operatorname{dim} X \leq 2$. 
Proof. Choose a perfect group $G$ containing integers (for example: $G=\left\{x_{1}, x_{2}, x_{3}\right.$, $\left.\left.x_{4} \mid x_{1}=\left[x_{2}, x_{3}\right], x_{2}=\left[x_{3}, x_{4}\right], x_{3}=\left[x_{4}, x_{1}\right], x_{4}=\left[x_{1}, x_{2}\right]\right\}\right)$ and notice that $G * \mathbf{z} G$ is perfect.

\section{Two-dimensional $L C^{1}$ COMPACTA}

It is well known that for compacta $X$ and $Y$ the equality

$$
\operatorname{dim}(X \times Y)=\operatorname{dim} X+\operatorname{dim} Y
$$

does not generally hold $\left[\mathrm{Bol}_{1}, \mathrm{Bor}_{1}, \mathrm{P}\right]$. Therefore it makes sense to study compacta such that the equality $(*)$ holds $\left[\mathrm{Ko}_{1}, \mathrm{Ko}_{2}\right]$. One of the known cases is of compacta $X$ and $Y$ which have the property $\Delta$ in the sense of K. Borsuk [Bor 2, p.178]. Since 2-dimensional ANR compacta have the property $\Delta$, the equality $(*$ ) holds (in fact, these spaces are dimensionally full-valued $\left[\mathrm{Ko}_{1}\right]$ ). Boltyanskii $\left[\mathrm{Bol}_{1}\right]$ (see also [Bor 1$]$ ) constructed 2-dimensional $L C^{0}$ compactum with $\operatorname{dim}(X \times X)=3$. Thus, to consider whether the equality $(*)$ holds in the class of 2-dimensional $L C^{1}$ compacta is natural. We shall solve this problem in the affirmative.

Definition. Recall that a space is called semilocally simply connected provided $X$ has a basis of open sets $U$ such that the inclusion induced homomorphism $\pi_{1}(U) \rightarrow$ $\pi_{1}(X)$ is trivial.

Theorem 4.1. Let $X$ be a locally connected and semilocally simply connected compactum of dimension $\geq 2$. Then, $\operatorname{dim}_{G} X>1$ for all groups $G \neq 1$.

Proof. Suppose $\operatorname{dim}_{G} X \leq 1$. Given $x \in X$ choose a connected and locally connected open neighborhood $U$ of $x$ such that $\pi_{1}(U) \rightarrow \pi_{1}(X)$ is trivial. Suppose $U$ contains a copy $S$ of the circle. Then, there is a non-trivial map $f: S \rightarrow K(G, 1)$ which extends to $F: X \rightarrow K(G, 1)$. This is a contradiction since $S \rightarrow X$ is null-homotopic. Thus, $\operatorname{dim} U \leq 1$ which implies $\operatorname{dim} X \leq 1$, a contradiction.

Corollary 4.2. Let $X$ be a two-dimensional, locally connected and semilocally simply connected compactum. Then, for any compactum $Y$

$$
\operatorname{dim}(X \times Y)=\operatorname{dim} X+\operatorname{dim} Y .
$$

Proof. It is proved in $\left[\mathrm{Bol}_{2}\right]$ (see also [Ku], Theorem 11 on p.22) that $\operatorname{dim}(X \times Y)=$ $\operatorname{dim} X+\operatorname{dim} Y$ for all compacta $Y$ is equivalent to $\operatorname{dim} X=\operatorname{dim}_{G} X$ for all abelian groups $G \neq 0$. Now, Corollary 4.2 follows from Theorem 4.1.

\section{REFERENCES}

[A] P.S.Alexandroff, Dimensionstheorie, ein Beitrag zur Geometrie der abgesehlossenen Mengen, Math. Ann. 106 (1932), 161-238.

[Bol 1 V.G.Boltyanskii, An example of a two-dimensional compactum whose topological square is three-dimensional, Dokl. Acad. Nauk SSSR 67 (1949), 597-599. (Russian) MR 11:45e

[Bol $\mathrm{B}_{2} \longrightarrow$, On dimensional full valuedness of compacta, Dokl. Acad. Nauk SSSR 67 (1949), 773-777. (Russian) MR 11:195j

[Bor 1 K.Borsuk, Concerning the cartesian product of Cantor-manifolds, Fund. Math. 37 (1951), 55-72. MR 14:72e

[Bor 2$]$, Theory of retracts, PWN, Warszaw, 1967. MR 35:7306 
[C] T.A.Chapman, Lectures on Hilbert cube manifolds, CBMS Regional Conference Series in Mathematics No.28, AMS, 1975, pp. 1-131. MR 54:11336

[Da 1 R.J.Daverman, Decompositions of manifolds, Academic Press, Orlando, 1986. MR 88a:57001

[Da 2$]$, Hereditarily aspherical compacta and cell-like maps, Topology Appl. 41 (1991), 247-254. MR 93b:54033

[D-D] R.J.Daverman and A.N. Dranishnikov, Cell-like maps and aspherical compacta (to appear).

[Dra] A.N.Dranishnikov, Homological dimension theory, Russian Math. Surveys 43(4) (1988), 11-63. MR 90e:55003

[D-R] A.N.Dranishnikov and D.Repovs, Cohomological dimension with respect to perfect groups, preprint (1991).

[Dy1] J.Dydak, The Whitehead and Smale theorems in shape theory, Dissert. Math. 156 (1979), 1-51. MR 80h:55008

[Dy 2$] \longrightarrow$ Cohomological dimension and metrizable spaces, Transactions of the Amer.Math. Soc. 337 (1993), 219-234. MR 93g:55001

[D-S 1 J.Dydak and J.Segal, Shape theory: An introduction, Lecture Notes in Math. 688, Springer Verlag, 1978, pp. 1-150. MR 80h:54020

[D-S $\left.{ }_{2}\right]$, Local n-connectivity of decomposition spaces, Topology and its Appl. 18 (1984), 43-58. MR 86a:54043

[En] R.Engelking, Dimension Theory, Math. Library, North-Holland, 1978. MR 58:2753b

[Hu] S.T.Hu, Theory of retracts, Wayne State University Press, 1965, pp. 1-234. MR 31:6202

[Ko1] Y.Kodama, On homotopically stable points and product spaces, Fund. Math. 44 (1957), 171-185. MR 20:279

$\left[\mathrm{Ko}_{2}\right] \quad$, Appendix to K. Nagami, Dimension theory, Academic Press, New York, 1970.

[Ku] V.I Kuz'minov, Homological dimension theory, Russian Math. Surveys 23 (1968), 1-45. MR 39:2158

[P] L.Pontryagin, Sur une hypothese fondamentale de la theorie de la dimension, C. R. Acad. Sci. Paris 190 (1930), 1105-1107.

[Sp] E.Spanier, Algebraic topology, McGraw-Hill, New York, 1966. MR 35:1007

[Wa] J.J.Walsh, Dimension, cohomological dimension, and cell-like mappings, Lecture Notes in Math. 870, Springer-Verlag, 1981, pp. 105-118. MR 83a:57021

[We] J.West, Open problems in infinite dimensional topology, Open Problems in Topology, North-Holland, 1990. CMP 91:03

[Wh] George W.Whitehead, Elements of homotopy theory, Springer-Verlag, New York, 1978. MR 80b:55001

Department of Mathematics, University of Tennessee, Knoxville, Tennessee 37996

E-mail address: dydak@math.utk.edu

Institute of Mathematics, University of Tsukuba, Tsukuba-shi, Ibaraki, 305, Japan

E-mail address: yokoi@sakura.cc.tsukuba.ac.jp 